Archives

40 | 2007

Dépendance(s)

\title{
Introduction
}

Dépendance(s) :circulation et usages d'un mot entre l'histoire et les sciences sociales

\section{Maurice Aymard}

\section{Q OpenEdition}

Journals

Édition électronique

URL : http://journals.openedition.org/ccrh/3331

DOI : $10.4000 /$ ccrh.3331

ISSN : $1760-7906$

Éditeur

Centre de recherches historiques - EHESS

Édition imprimée

Date de publication : 25 avril 2007

Pagination : 7-14

ISSN : 0990-9141

\section{Référence électronique}

Maurice Aymard, «Introduction », Les Cahiers du Centre de Recherches Historiques [En ligne], 40 | 2007,

mis en ligne le 15 novembre 2011, consulté le 01 mai 2019. URL : http://journals.openedition.org/ ccrh/3331 ; DOl : 10.4000/ccrh.3331

Ce document a été généré automatiquement le 1 mai 2019.

Article L.111-1 du Code de la propriété intellectuelle. 


\title{
Introduction
}

\author{
Dépendance(s) :circulation et usages d'un mot entre l'histoire et les \\ sciences sociales
}

Maurice Aymard

1 Pourquoi nous poser aujourd'hui la question de la dépendance ? Pourquoi, et en fait aussi comment? Recevant le message de Gérard Béaur, en mai 2005, j'avais aussitôt pensé à deux livres, et à un débat. Le débat avait été animé dans les années soixante et soixantedix par un ensemble international d'économistes et de sociologues qui, à propos surtout de l'Amérique Latine, avaient érigé la dépendance en théorie critique du développement. Pour ces derniers, dont m'avaient rapproché à l'époque mes recherches sur la Sicile à l'époque moderne (où il était tentant de lire les origines du développement inégal en Europe), je n'avais que l'embarras du choix : Arghiri Emmanuel (L'échange inégal) ${ }^{1}$, André Gunder Frank et Samir Amin (L'accumulation dépendante. Sociétés précapitalistes et capitalisme $^{2}$ et Fernando Henrique Cardoso, auteur, avec Enzo Faletto,de Dépendance et développement ${ }^{3}$. Mais ce débat, qui appartient aujourd'hui au passé, a été victime à la fois de la globalisation et de l'éclatement du tiers-monde, avec l'émergence non plus de simples petits dragons ou tigres, mais de grands pays, destinés, nous dit-on, à devenir les grandes puissances du XXI siècle : au premier rang d'entre eux, la Chine, l'Inde, le Brésil, et peut-être l'Afrique du Sud.

2 Les deux livres me semblaient en revanche permettre de mieux cadrer le problème que nous avaient posé Gérard Béaur et Bernard Vincent. Le premier : La Société féodale ${ }^{4}$ de Marc Bloch, un classique pour tous les historiens. Le second : La dépendance. Esquisse d'un portrait du dépendant ${ }^{5}$ d'Albert Memmi, prolongé et développé près de vingt ans plus tard par Le buveur et l'amoureux. Le prix de la dépendance ${ }^{6}$. D'un côté un ouvrage qui décrivait l'organisation globale d'une civilisation qui avait présidé à la naissance de l'Europe médiévale, et proposait la dépendance comme clef de lecture générale, car institutionnalisée, d'une organisation d'ensemble de la société. De l'autre l'essai d'un écrivain et philosophe qui interrogeait et analysait la société contemporaine pour y mettre en évidence l'omniprésence de la relation de dépendance, aussi bien dans nos comportements quotidiens et les situations sociales concrètes observables autour de nous que dans les représentations que nous en proposent les romans, les films et les autres 
modèles idéaux qui orientent nos décisions et nos actions, nos pensées et nos rêves. Une omniprésence, une universalité d'autant plus surprenantes que cette dépendance n'y prend jamais le visage d'une institution: car notre société se pense, se dit et se croit, le plus sincèrement du monde, fondée sur l'égalité entre les personnes, sur l'autonomie des individus, sur le respect de l'autre et sur la dénonciation des dominations officielles ou clandestines, ouvertes ou souterraines.

3 L'historien Marc Bloch se situe du côté du collectif et de l'institution qui s'impose à tous et modèle les façons de penser et d'agir des individus. Il ne prend même pas la peine de justifier ou de définir ces "liens de dépendance », dont il fait le titre de son premier volume. Il se contente d'en résumer la conclusion au début du second,

un réseau de liens de dépendance, tissant ses fils du haut en bas de l'échelle humaine, donna à la civilisation de la féodalité européenne son empreinte la plus originale [...]. Jamais [...] les destinées individuelles n'avaient été réglées exclusivement par ces rapports de proche sujétion ou d'immédiat commandement.

Tout comme il faut être citoyen d'un État dans le monde contemporain, il faut, pour exister dans la société féodale, être l'homme d'un autre homme : son dépendant reconnu, accepté et affirmé. Ces liens d'homme à homme englobent les liens du sang, la vassalité et le fief, mais concernent aussi les classes inférieures, dont la majorité rurale est intégrée dans le cadre de la seigneurie, et dont les formes et les transformations de la servitude règlent aussi bien la vie quotidienne que le destin à long terme. Ils orientent également (cf. t. 2) «les classes et le gouvernement des hommes» (noblesse, clergé, justices, pouvoirs " politiques »).

5 À l'opposé de Marc Bloch, en apparence au moins, Albert Memmi joue sur le double registre de l'individuel et de l'universel :

À la question : qui est dépendant? Je suis convaincu, qu'on doit répondre "tout le monde". Chacun à sa manière, certes, inégalement, relativement à un ou plusieurs objets, d'une manière dynamique et variable selon les conjonctures. Mais ce portrait de l'homme dépendant qui est ici esquissé, pourrait être, judicieusement retouché selon les cas, celui de n'importe qui. L'homme dépendant, enfin, est encore l'une des figures les plus courantes et les plus indiscutables de l'universelle humanité; au même titre que celle de l'homme dominant ou celle de l'homme dominé. Et souvent, c'est la même, vue sous un autre éclairage, car si les hommes se dominent fréquemment les uns les autres, ils ont au moins autant besoin les uns des autres?

Le paradoxe est que nous nous trouvons aujourd'hui, quand nous nous interrogeons en historiens sur la ou les dépendance(s), dans une situation plus proche de celle d'Albert Memmi que de celle de Marc Bloch.

7 La dépendance intéressait en effet Marc Bloch par sa dimension institutionnelle totale, qui modelait et résumait toutes les autres institutions de la société féodale. Lorsqu'il écrit «Jamais [...] les destinées individuelles n'avaient été réglées exclusivement par ces rapports de proche sujétion ou d'immédiat commandement ", il pense sans doute, par opposition, aux relations de clientèle dans le monde romain : celles-ci lient à un patron aussi bien des hommes libres que des affranchis qui, après leur libération, doivent à leur ancien maître (dont en général ils portent le nom) une série d'obligations. Mais ces liens codifiés (plus qu'institutionnalisés au sens "public» du terme) de dépendance qui conciliaient l'égalité statutaire de l'homme libre et l'inégalité de fait, acceptée, recherchée ou imposée, et qui engageaient aussi bien le patron que le client, ne se sont jamais étendus à la totalité de la société romaine. Cette dépendance particulière 
coexistait avec d'autres, de nature différente (par exemple, dans les campagnes, celles des paysans vis-à-vis des propriétaires, avec la généralisation du "colonat»), et avec des relations interpersonnelles, impossibles à analyser en termes de dépendance.

Ce qui intéresse Albert Memmi, c'est au contraire la dimension à la fois générale et (sauf exception) non ou faiblement institutionnelle d'une relation plus ou moins librement consentie, mais toujours aussi plus ou moins acceptée et souvent recherchée et intériorisée. Cette relation est plus complexe qu'il n'y paraît, car elle prend à ses yeux une forme toujours triangulaire. «La dépendance est une relation trinitaire : deux partenaires et un objet », qu'il propose de nommer « le dépendant », « le pourvoyeur », et « l'objet de pourvoyance » Et «il sera toujours instructif de se poser les trois questions: «Qui est dépendant? De qui ? Et de quoi ? $»^{8}$. Quel que soit le respect que nous pouvons avoir pour l'auteur du Portrait d'un colonisé, cette revendication d'une omniprésence de la relation de dépendance a de quoi nous irriter, car nous sommes spontanément portés à penser que si la dépendance est partout, elle n'est nulle part, et que, si elle peut se retrouver en tous temps et en tous lieux, elle perd de ce fait toute possibilité de s'inscrire dans une histoire. Nous nous méfions, professionnellement, des catégories universelles, les individus n'existent à nos yeux que replacés dans des situations sociales concrètes, et l'utilisation des personnages des œuvres culturelles, nés de l'imagination d'un "créateur ", écrivain ou cinéaste, est soumise à des règles de prudence visant à nous protéger contre le triple effet de réalité, d'identification et de distanciation que ces œuvres cherchent précisément à produire en nous. Mais le programme qui nous réunit le montre, l'utilisation du terme de dépendance par les historiens au cours de ces deux ou trois dernières décennies, et la réflexion critique qui nous réunit aujourd'hui sur les implications, les finalités, les modalités et la légitimité de ces usages, rejoignent sur plus d'un point, sans pour autant s'en inspirer directement, car les historiens ne le citent guère, les pistes tracées par Albert Memmi.

9 Pour la majorité sinon la totalité d'entre nous, ce qui nous attire et retient notre attention dans les relations ou situations de dépendance, se situe sur un double registre.

Le premier est le fait qu'elles viennent enrichir et diversifier une lecture qui se voulait « objective » des hiérarchies sociales tant en termes d'ordres qu'en termes de classe : les premiers privilégiaient la naissance, les fonctions exercées, les positions acquises et transmises, les seconds mobilisaient au contraire un éventail de plus en plus large d'indicateurs statistiques : propriété du sol, niveau des revenus, valeur des dots et des patrimoines, niveau culturel, professions exercées, etc.

11 Le second correspond moins au modèle proposé par Marc Bloch pour la société féodale, où la dépendance constituait le cœur du système, qu'à leur caractère au contraire à la fois non institutionnel ou faiblement institutionnalisé: l'important est que, même si ces relations et ces situations ont une origine institutionnelle, l'institution ne suffit pas à les expliquer. Et le fait que profon-dément acceptées, intériorisées, recherchées même, elles fonctionnent comme des modèles qui assurent leur propre reproduction et leur résistance au temps. Elles peuvent se mettre en place indépendamment de toute institution. Et il ne suffit pas d'abolir les institutions et les normes qui avaient pu leur servir de cadre ou de soutien pour les faire disparaître : elles se perpétuent pendant des générations, et continuent à servir de référence pour la majorité des acteurs. Une permanence que ne suffisent pas à expliquer les causes économiques, sociales ou culturelles le plus souvent invoquées. De même que l'affranchissement légalement décidé n'a suffi à abolir ni la réalité ni le souvenir de l'esclavage, tout l'arsenal législatif visant à 
assurer l'égalité des femmes et des hommes peine et tarde à transformer les situations concrètes. Il en est de même pour les inégalités entre aînés et cadets, ou entre garçons et filles dans les successions, qui créent fréquemment des situations de dépendance de fait entre parents et enfants, d'une part, et entre enfants de sexe différent et/ou de rang inégal: nous savons qu'elles ont résisté tout au long du XIXe siècle, et peut-être plus longtemps encore, aux décisions de la Révolution et aux règles du Code Civil. D'où l'accent que nous avons été conduits à mettre sur les pratiques et l'analyse micro-historique des comportements des individus, des familles et des groupes : nous leur demandons aussi la clef de cette mémoire très particulière du social, que le concept de « survivance » ne suffit ni à décrire ni à expliquer.

Les exemples donnés par Memmi dans la première partie de son livre permettent de noter d'indiscutables convergences avec les intérêts de l'histoire sociale récente. Couples sociaux: "colonisateur-colonisé, homme-femme, blanc-noir, maître-domestique ». Rapports amoureux et rapports parents-enfants. Pratiques magiques et rituels de possession et d'exorcisme. Consommations, comme celles du tabac ou de l'alcool conçues comme étant des " comportements collectifs régis par des valeurs ». Figures féminines qui peuplent notre imaginaire collectif, de la patrie et de notre sainte mère l'Église à l'Université comme alma mater. Ou, de même, figures de la maison et de la famille, messianismes, etc. Autant de thèmes qui ont retenu, au cours de ces dernières décennies l'attention des historiens, et pour l'analyse desquels ils ont retrouvé sur leur route, dans plus d'un cas sinon dans tous, le terme de dépendance. Celui-ci leur est apparu le plus adéquat - car moins réducteur et plus riche de significations que celui de domination pour décrire des systèmes de relations interpersonnelles hiérarchisées et de représentations de ces relations.

13 L'utilisation d'un mot n'étant jamais innocente, on remarquera qu'une fois encore les historiens se sont abstenus d'en donner une définition rigoureuse préalable. Et ceci pour au moins deux raisons :

- sa souplesse d'utilisation : l'absence d'une définition rigoureuse donne la possibilité de regrouper des situations d'inégalité ou d'infériorité sociale, économique ou culturelle, individuelles ou collectives, mais toujours extrêmement diversifiées, qui avaient en commun de déborder très largement le champ du statut juridique ou institutionnel, et donc d'exister et de se reproduire indépendamment de tout statut institué, et de pouvoir ainsi précéder son éventuelle apparition, se perpétuer après son abolition ou sa modification, ou même éviter de l'officialiser. Ce qui permettait de jouer le fait contre le droit, le vécu contre l'institué, les pratiques contre les normes, en mettant en cause des durées plus longues et des espaces sociaux plus étendus, des contaminations, des continuités et des résurgences. Et d'effectuer, des premiers aux seconds termes de ces couples de mots, de multiples allers et retours.

- le mot avait l'avantage d'être dans l'air du temps, et largement utilisé dans les autres sciences sociales. Les historiens pouvaient ainsi emprunter avec le mot toute une série de valences plus ou moins métaphoriques. Mais, il faut le reconnaître aussi, ils n'ont pas cherché à y mettre de l'ordre, soit pour maintenir au mot de dépendance son statut de terme de leur langue savante, soit pour filtrer les usages des autres sciences sociales qu'ils pouvaient ou souhaitaient reprendre à leur compte.

Résultat: le terme a cessé d'être associé à l'histoire. Un sondage rapide dans Google permet d'y retrouver, dans plus d'une quinzaine de pages, l'appel à cette rencontre du Centre de recherches historiques, mais guère d'autre référence à l'histoire. Le classement 
des sujets Rameau de la BNF identifie les sous-catégories de dépendance alimentaire (cf. aliments, approvisionnement), bio-logique et physique (cf. dépendance, physiologie), linguistique (cf. grammaire de dépendance), psychologique et politique. Mais pas l'histoire, et pas davantage l'économie, malgré la théorie de la dépendance (point de passage obligé des analyses du développement inégal dans les années soixante-soixantedix), et alors que dans les différentes encyclopédies de sciences sociales, elle est la seule à faire l'objet d'une entrée spécifique (Encyclopedia of Sociology, Macmillan, 1992). Ou malgré encore la formalisation récente de la "path dependency " (dépendance temporelle), inspirée de l'exemple des systèmes techniques et fondée sur l'analyse comparée des coûts de transaction. Il en est de même dans les encyclopédies disponibles sur la toile: Wikipedia distingue ainsi dépendance en architecture, en géographie, en informatique ( «le fait d'avoir besoin d'un logiciel donné pour en utiliser un autre, ou le fait que la valeur de $\mathrm{X}$ influe sur le comportement de $\mathrm{Y} »)$, en toxicologie (de laquelle est rapprochée la dépendance sexuelle ou celle à internet), en psychologie (dépendance affective) et enfin en microbiologie ( terme équivalent à système poison-antidote, ou à système de mort programmée, ou à système de post segregational killing, pour désigner des éléments identifiés sur des plasmides à faible nombre de copies »).

Il vaudra dans ces conditions la peine de suivre le chemin parcouru depuis Furetière, qui distinguait trois significations fondamentales de dépendance :

1. sujétion, infériorité (antonyme : liberté) : les références
sont nombreuses sur ce point à l'organisation féodale
et ecclésiastique, fondée sur un système de
hiérarchie, mais les exemples concernent les choses
(un fief, un prieuré) mais non les personnes
2. connexité, suite nécessaire : «toutes les propositions de
géométrie ont une suite de dépendance les unes des
autres "
3. ce qui fait partie d'un tout : « ce hameau est la
dépendance de cette paroisse ».

Tout se passe donc comme si les sciences sociales avaient laissé à la langue naturelle les significations 1 et 3 (l'histoire utilisant surtout la première), pour, d'un côté, s'approprier la seconde, celle de la suite nécessaire (cf., comme pour l'informatique, dans le cas de la linguistique, les grammaires de dépendance ou la phonologie de la dépendance) et, de l'autre, en développer deux nouvelles. La première, qui dérive en partie de la première de ces trois significations, correspond à l'insuffisance des quantités disponibles pour faire face à un besoin ressenti (dépendance alimentaire, ou énergétique), ou, de manière plus générale, à la nécessité de recourir à d'autres dans une situation de perte d'autonomie (dépendance des vieillards, des grands malades, de telle ou telle catégorie de handicapés). La seconde au contraire, radicalement nouvelle, est celle qu'ont privilégiées la médecine et la psychologie (et la psychanalyse) : la première s'en sert pour désigner « un état où, malgré une conscience aiguë des problèmes liés à une consommation abusive, l'individu n'est plus capable de contrôler sa consommation "; la seconde, à propos de la dépendance affective, pour désigner « un besoin général et excessif d'être pris en charge conduisant à un comportement et à une angoisse de séparation ». Cette signification correspond à ce que l'anglais traduit le plus souvent par "addiction", quand il s'agit de désigner les accoutumances aux drogues, à l'alcool, au tabac, au téléphone portable, et à d'autres formes de comportement ou de consommation étiquetées comme « déviantes ». 
17 L'historien se trouve donc placé devant au moins trois pistes, entre lesquelles, même si rien ne le force à choisir, son "habitus » le pousse à suivre de préférence les deux premières

- celle de l'extension du territoire de l'historien: étendre à de nouveaux domaines l'utilisation du terme de dépendance ; explorer de façon systématique la multiplicité, la complexité et parfois aussi les contradictions des hiérarchies qui établissent et formalisent des relations à la fois d'inégalité et de complémentarité entre les individus et les groupes; approfondir notamment l'étude des relations interpersonnelles et la transformation des individus en sujets d'une histoire qui intègre à la fois la modification au cours d'une vie des positions relatives des acteurs, le caractère aléatoire (et donc considéré comme libre) de leurs décisions individuelles dont aucune n'est totalement prédéterminée, et les phénomènes de réciprocité ou de renversement par les dépendants de leur situation d'infériorité.

- celle de l'histoire des usages du mot, de ses champs d'application, des glissements sémantiques dont il a pu faire l'objet. On notera ainsi chez Montesquieu, dans L'Esprit des Lois, cette affirmation très "machiste", mais que bien d'autres auteurs du XVIII siècle auraient pu écrire, sous une forme à peine différente, pour défendre la validité objective d'une sujétion très ancienne (alors qu'il condamne par ailleurs l'esclavage dans les termes d'une rare efficacité dans leur concision) :

La raison n'est jamais accompagnée par la beauté chez la femme. Lorsque la beauté voudrait dominer, la raison lui empêche, lorsque la raison pourrait dominer, la beauté a disparu. Les femmes doivent rester dépendantes.

19 Ou encore l'on enregistrera par exemple l'entrée du mot de dépendance dans le vocabulaire de l'économie.

La troisième piste, plus difficile et moins familière aux historiens, viserait à tenter d'aller plus loin, et à s'appuyer sur les résultats établis en suivant les deux premières pour interroger le terme même de dépendance, en proposer des usages plus rigoureux, le distinguer de ses différents synonymes et antonymes. Dans la conclusion de son enquête, Albert Memmi propose ainsi de distinguer les quatre mots qu'il avait précédemment utilisés, et dont il propose de faire deux couples distincts. D'un côté, domination (point de vue du dominant: «l'ensemble des contraintes imposées par le dominant sur le dominé ») et sujétion (point de vue du dominé ( «l'ensemble des réponses, actives ou passives, du dominé aux agressions du dominant»). De l'autre, pourvoyance («ce qui répond à l'attente du dépendant ») et dépendance (« relation contraignante, plus ou moins acceptée, avec un être, un objet, un groupe ou une institution, réels ou idéels, et qui relève de la satisfaction d'un besoin »). Si l'on compare domination et dépendance,

ce qui les différencie l'emporte sur ce qui les rapproche : en un mot, le dépendant consent plus ou moins à son aliénation, le dominé, non. On peut, certes, être à la fois dépendant et dominé, mais cette coïncidence n'est ni automatique ni nécessaire. La dépendance ne se confond pas avec la sujétion $[. . .]^{9}$.

21 Une telle démarche nous permettrait, en jouant sur les termes de domination, de sujétion, de dépendance, et d'autres encore, de mieux prendre en compte les situations concrètes et les réactions des dominés et des dépendants. De renouveler en la nuançant l'étude des groupes et des classes que l'on aimait, il y a trente ans, référence à Gramsci obligeait, à définir comme "subalternes». Et d'analyser leurs stratégies de réappropriation et de renversement, réel ou symbolique, de cette relation, et celles que développent en sens contraire les « dominants » et les « pourvoyants » pour les maintenir 
ou, mieux encore, les renouveler de l'intérieur, en suscitant de nouveaux besoins et de nouveaux moyens pour y répondre. La même démarche viserait aussi à croiser étroitement, d'un côté, l'étude des trajectoires individuelles et celle des situations collectives, et, de l'autre, celle des réalités et celle des représentations, non pas pour les opposer les unes aux autres, mais pour mieux comprendre leus interférences et leurs interactions. En se plaçant d'entrée de jeu sous le signe d'une complexité affirmée et revendiquée comme telle, elle se situerait dans le droit fil des orientations actuelles de la recherche dans cette science sociale très particulière qu'est l'histoire.

\section{NOTES}

1. Arghiri Emmanuel, L'échange inégal, Paris, Maspéro, 1969.

2. André Gunder Frank, Samir Amin, L'accumulation dépendante. Sociétés précapitalistes et capitalisme, Paris, Éditions Anthropos, 1978 (trad. franç. Eddy Trèves).

3. Fernando Henrique Cardoso, Enzo Faletto, Dépendances et Développement, Paris, PUF, 1978 (trad. franç. Annie Morvan).

4. Marc Bloch, La Société féodale,t. 1: La Formation des liens de dépendance, t. 2 : Les classes et le gouvernement des hommes Paris, Albin Michel, 1939.

5. Albert Memmi, La Dépendance : esquisse pour un portrait du dépendant, Paris, Gallimard, 1979.

6. Id. Le buveur et l'amoureux : le prix de la dépendance, Paris, Arléa, 1998.

7. Albert Memmi, La Dépendance [...], op. cit., p. 206.

8. Ibid., p. 18.

9. Albert Memmi, La Dépendance [...], op. cit., p. 198.

\section{AUTEUR}

\section{MAURICE AYMARD}

MSH Paris 